\title{
Recent Developments in Heavy-Quarkonium Phenomenology
}

\author{
Hee Sok Chung ${ }^{\mathrm{a}}$, Jungil Lee ${ }^{\mathrm{a} *}$ and Chaehyun $\mathrm{Yu}^{\mathrm{a}}$ \\ ${ }^{a}$ Department of Physics, Korea University, Seoul 136-701, Korea
}

\begin{abstract}
We review recent developments in heavy-quarkonium phenomenology within the nonrelativistic QCD factorization approach. Main issues we consider in this work include the polarization of prompt $J / \psi$ at the Fermilab Tevatron and the large relativistic and QCD corrections to double-charmonium production at the $B$ factories. We also consider inclusive charm production in bottomonia decays.
\end{abstract}

\section{Introduction}

For more than a decade, the nonrelativistic quantum chromodynamics (NRQCD) factorization approach [1] has been employed to predict various measurables in heavy-quarkonium phenomenology. In this approach, rates for production or decay of a heavy quarkonium that involve creation or annihilation of a heavy-quarkantiquark $(Q \bar{Q})$ pair are expressed in linear combinations of NRQCD matrix elements, which reflect the long-distance nature of the heavy quarkonium. Corresponding short-distance coefficients are insensitive to the long-distance interaction and calculable perturbatively. As the first application of the factorization theorem for the decays, the light-hadronic and electromagnetic decay rates of the $P$-wave heavy quarkonia were factorized [2]. Explicit infrared singularities appearing in order- $\alpha_{s}^{3}$ color-singlet-model predictions for the light-hadronic decays of $P$-wave heavy quarkonia $[3,4]$ became to be identified as those of the next-to-leading-order (NLO) corrections to the long-distance color-octet NRQCD matrix elements that mix with the color-singlet NRQCD matrix elements at this order.

Soon after the resolution of the infrared problem in the $P$-wave quarkonium decay, $\psi(2 S)$ anomaly was reported: the transversemomentum $\left(p_{T}\right)$ distributions for prompt $\psi(2 S)$ and $J / \psi$ measured by the CDF Collaboration at

\footnotetext{
*This work was supported by the Korea Science and Engineering Foundation (KOSEF) funded by the Korea government (MEST) under grant No. R01-2008-000-10378-0.
}

the Fermilab Tevatron were greater than theoretical predictions of the color-singlet model by orders of magnitude at large $p_{T}[5,6]$. Based on the idea of gluon-fragmentation dominance of quarkonium production at large $p_{T}[7]$, the puzzle was resolved by introducing the color-octet $S$-wave spin-triplet $\left[Q \bar{Q}_{8}\left({ }^{3} S_{1}\right)\right]$ contribution to the gluon fragmentation into an $S$-wave spin-triplet heavy quarkonium [8]. The dominance of the color-octet mechanism in large- $p_{T}$ prompt $\psi(2 S)$ production led to the prediction that the prompt $\psi(2 S)$ must be transversely polarized at large $p_{T}[9,10]$. Unfortunately, the predictions of the polarizations of prompt $\psi(2 S)[11,12]$ and prompt $J / \psi[13,14]$ were disfavored by the CDF measurement at Run I of the Fermilab Tevatron [15]. For more details, we refer the readers to Refs. $[16,17]$. This problem has been a serious challenge to heavy-quarkonium theory.

Another interesting problem in quarkonium phenomenology that has not been resolved until very recently is regarding the cross section for the exclusive $J / \psi+\eta_{c}$ production in $e^{+} e^{-}$ annihilation at $B$ factories. The cross section measured by the Belle Collaboration [18] was greater than theoretical predictions at leading order (LO) in $\alpha_{s}$ and $v$ by an order of magnitude $[19,20]$. The BABAR Collaboration also observed a large cross section [21] and subsequent studies $[22,23]$ of the Belle Collaboration revealed that the large discrepancy between theory and data is not originated from the contamination of other signals [24-26]. On the other hand, there were arguments that an appropri- 
ate choice of light-cone distribution amplitude may resolve the problem $[27,28]$, which were confronted with an opposing argument that, after separating the short-distance contribution from the long-distance light-cone distribution amplitude, the light-cone method yields a result equivalent to that of NRQCD [29]. Very recently, large relativistic corrections [30,31] in combination with one-loop QCD corrections $[32,33]$ filled the gap within errors [34].

In this work we review recent developments in heavy-quarkonium phenomenology within the nonrelativistic QCD factorization approach, focusing on the following three selected topics. Issues regarding the polarization of prompt $J / \psi$ at the Fermilab Tevatron and future opportunities to resolve the problem at the CERN LHC are presented in Sec. 2. The discrepancy between the LO predictions and the empirical cross sections for $e^{+} e^{-} \rightarrow J / \psi+\eta_{c}$ measured at the $B$ factories has been a long-standing puzzle. In Sec. 3 we briefly review the problem and describe a recently developed method of resumming a class of relativistic corrections to all orders in the heavy-quark velocity $v$, which played a crucial role in resolving the $e^{+} e^{-} \rightarrow J / \psi+\eta_{c}$ problem in conjunction with the relative-order- $\alpha_{s}$ corrections. As the last topic, inclusive charm productions in bottomonia decay being analyzed by the CLEO Collaboration and corresponding theoretical predictions are reviewed in Sec. 4 which is followed by conclusions in Sec. 5.

\section{Polarization of prompt $J / \psi$}

The discrepancy between the theoretical prediction and the CDF measurement at Run I of the Fermilab Tevatron for the prompt $S$-wave spin-triplet charmonium produced at large $p_{T}$ was embarrassing. Gluon fragmentation has the kinematic enhancement factor of $p_{T}^{2} / m_{J / \psi}^{2}$ which dominates over the suppression factor $\alpha_{s}^{2}\left(p_{T}\right)$ for the coupling compared to the gluon-fusion diagrams at sufficiently large $p_{T}[7]$. In comparison with the gluon fragmentation into the colorsinglet $Q \bar{Q}$ pair $\left[Q \bar{Q}_{1}\left({ }^{3} S_{1}\right)\right]$, the $Q \bar{Q}_{8}\left({ }^{3} S_{1}\right)$ contribution has a less severe suppression factor of order $v^{4}$ which accounts for the spin-non-flipping long- distance transition from the color-octet to the color-singlet state, while the suppression factor $\alpha_{s}^{2}\left(p_{T}\right)$ in the color-singlet fragmentation channel is for the short-distance coefficient [8]. If the gluon fragmentation into the $Q \bar{Q}_{8}\left({ }^{3} S_{1}\right)$ pair is the dominant contribution to the prompt $J / \psi$ and $\psi(2 S)$ production at large $p_{T}$, then the invariant mass for the fragmenting virtual gluon is negligible compared to the typical scale $p_{T}$ of the short-distance process. Hence, longitudinally polarized virtual-gluon contribution must be suppressed compared to the transverse contribution with a multiplicative scaling factor of $m_{J / \psi}^{2} / p_{T}^{2}[9,10]$. Because the long-distance transition from $Q \bar{Q}_{8}\left({ }^{3} S_{1}\right)$ to $Q \bar{Q}_{1}\left({ }^{3} S_{1}\right)$ is dominated by spin-non-flipping chromoelectric dipole transitions, prompt $\psi(2 S)$ must be transversely polarized at large $p_{T}$. This is also supported by a lattice computation of the color-octet NRQCD matrix element involving the spin-flipping interaction for the decay [35]. On the contrary, the predictions for the $\psi(2 S)$ polarization [11-13] were disfavored by the CDF data at both Run I and Run II of the Fermilab Tevatron $[15,36]$.

In a similar manner as $\psi(2 S)$, one can expect that the prompt $J / \psi$, which has much larger statistics than $\psi(2 S)$, produced at large $p_{T}$ must be transversely polarized. However, prompt $J / \psi$ suffers from feeddowns from higher resonances such as $\psi(2 S)$ and $\chi_{c J}$. At the Tevatron, the feeddowns from $\psi(2 S)$ and $\chi_{c J}$ are about $10 \%$ and $30 \%$, respectively. The contribution from $\chi_{c J}$ dilutes the polarization and that from $\psi(2 S)$ is dominantly spin preserving $[13,14]$. Although feeddown from higher resonances makes the prediction for the prompt $J / \psi$ less dramatic than $\psi(2 S)$, the tendency of rising transverse polarization as $p_{T}$ increases is the same as that for $\psi(2 S)[13,14]$. Again, this expectation was confronted with the CDF data at both Run I and Run II of the Fermilab Tevatron $[15,36]$. One may suspect any flaws in the theoretical predictions. The prediction for the prompt $J / \psi$ polarization in Ref. [13,14] was at LO in $\alpha_{s}$ and $v$. One may expect that the inclusion of real gluon emission and higher-order contributions in $v$ that flip spin may dilute the polarization even if they 
are suppressed based the velocity-scaling rules of NRQCD. One may also suspect that the dominance of gluon fragmentation has not yet been achieved in the $p_{T}$ range accessible at the Fermilab Tevatron. At the same time, it is fair to notice that the discrepancy between the Run I and Run II data of the CDF Collaboration is not clearly understood, yet $[15,36]$.

Theoretical prediction for the $\Upsilon(n S)$ produced at the Fermilab Tevatron was not dramatic as the $S$-wave spin-triplet charmonium state because the available range of $p_{T}$ does not reach the region where gluon fragmentation dominates [37]. Theoretical uncertainties were large because of the large errors in various NRQCD matrix elements of bottomonia [38]. The CDF measurement of the $\Upsilon(n S)$ polarization [39] was consistent with the prediction within errors [37]. Around the time when the prompt $J / \psi$ polarization measurement of the CDF Collaboration was announced, the E866 Collaboration reported that $\Upsilon(1 S)$ is almost unpolarized while the samples for $\Upsilon(2 S)+\Upsilon(3 S)$ are almost transversely polarized [40] in their fixed-target experiment. This shows that the polarization of $\Upsilon(1 S)$, that has many channels of feeddown from higher resonances through $P$-wave states, is diluted compared to $\Upsilon(2 S)$ and $\Upsilon(3 S)$ states. Similar tendency has been observed by the D0 Collaboration at Run II of the Tevatron [41]. The D0 result for $\Upsilon(1 S)$ shows that $\Upsilon(1 S)$ is longitudinally polarized at most of $p_{T}$ regions below about $15 \mathrm{GeV}$. This may be due to the feeddown effect through $P$-wave resonances. However, transverse contribution increases continuously from the maximally longitudinal region around $2-5 \mathrm{GeV}$ as $p_{T}$ increases. The D0 result for $\Upsilon(2 S)$, which has less feeddown effect than $\Upsilon(1 S)$ shows that the rise of transverse polarization is faster and stronger than that of $\Upsilon(1 S)$. This indicates that polarization analysis for $\Upsilon(n S)$ at the CERN LHC, where accessible $p_{T}$ may be large enough to approach gluon-fragmentation-dominance region for $\Upsilon(n S)$ production, may reveal transversely polarized spin-triplet $S$-wave bottomonia. In comparison with the charmonium counterparts, $\Upsilon(n S)$ is more nonrelativistic. As a result, it will suffer less from the contamination of longitudinal con- tributions of higher orders in $\alpha_{s}$ and $v$.

We list recent theoretical attempts that have been made to improve the NRQCD predictions for the inclusive heavy-quarkonium production at hadron colliders. The QCD NLO corrections to the color-singlet channel to the gluon-fusion diagrams, $g g \rightarrow Q \bar{Q}_{1}\left({ }^{3} S_{1}\right)+g$, that contributes to the ${ }^{3} S_{1}$ quarkonium production at hadron colliders were recently computed [42]. It was reported that the NLO corrections are large and modify the expectations for the total cross sections and the distribution in the transverse momentum. This is a signal that the NRQCD matrix element for the long-distance process $Q \bar{Q}_{8}\left({ }^{3} S_{1}\right) \rightarrow$ $J / \psi+X$ may be smaller than that estimated before, resulting in less dramatic increase of transverse polarization of prompt $J / \psi$. In Ref. [43], the contribution of the following specific channels, $g g \rightarrow Q \bar{Q}_{1}\left({ }^{3} S_{1}\right)+Q \bar{Q}$, where $Q$ is $c$ or $b$, were calculated. The authors reported that, in those channels, fragmentation approximation underestimates the cross section in the kinematical region accessible at the Fermilab Tevatron. In addition, the polarization of both $J / \psi$ and $\Upsilon$ produced through above channels are unpolarized. In Ref. [44], the authors update the theoretical predictions for hadroproduction of direct $\Upsilon$. They reported that the QCD NLO corrections to the color-singlet contribution significantly enhances the theoretical prediction for the cross section at large $p_{T}$ and substantially affect the polarization of the $\Upsilon$ [44]. Because the measured polarizations are for the inclusive $\Upsilon$ that contain various feeddowns, one cannot make a direct comparison with the available data for the inclusive $\Upsilon(n S)$. Further theoretical predictions for partial contributions to the inclusive charmonium production at hadron colliders are also available [45-47].

\section{Relativistic corrections to quarkonium processes}

In general, because of clean initial states, processes for quarkonium production in $e^{+} e^{-}$annihilation may have less theoretical uncertainties than hadroproduction. Especially, the exclusive double-charmonium production from $e^{+} e^{-}$annihilation into a virtual photon should be described 
relatively with ease because the color-singlet contribution in NRQCD is absolutely the dominant production mechanism. For the exclusive process $e^{+} e^{-} \rightarrow J / \psi+\eta_{c}$, only a single long-distance color-singlet NRQCD matrix element, $\left\langle\mathcal{O}_{1}\right\rangle_{J / \psi}$, involves, once we impose the approximate heavyquark spin symmetry of NRQCD. $\left\langle\mathcal{O}_{1}\right\rangle_{J / \psi}$ is the most accurately known NRQCD matrix element, which is determined from $\Gamma\left[J / \psi \rightarrow e^{+} e^{-}\right]$.

However, the cross section first measured by the Belle Collaboration, which is about $33 \mathrm{fb}$ [18], was greater than the NRQCD predictions $[19,20]$ at LO in both $\alpha_{s}$ and $v$ by an order of magnitude. Later the Belle Collaboration updated the value as $25.6 \pm 2.8 \pm 3.4 \mathrm{fb}$ [22] and the BABAR Collaboration also reported a large cross section of $17.6 \pm 2.8 \pm 2.1 \mathrm{fb}$ [21], where the values include the branching fraction of the system recoiling against the $J / \psi$ into two or more charged tracks. The theoretical predictions were $3.78 \pm 1.26 \mathrm{fb}[19]$ and $5.5 \mathrm{fb}$ [20], where the difference depends on the choice of input parameters. The prediction in Ref. [19] also includes QED contributions that make a constructive interference of about $30 \%$ of the total cross section for the QCD process. As was described earlier, proposals that the signal may include final states other than $J / \psi+\eta_{c}[24-26]$ were disfavored by an updated analysis by the Belle Collaboration $[22,23]$. And the light-cone method reproduced the NRQCD prediction [29]. This puzzle has been a serious challenge to quarkonium phenomenology.

When the first theoretical prediction was made, it was reported that there may be huge relativistic corrections to the LO prediction [19]. Unfortunately, there was no reliable way available to estimate the NRQCD matrix element involving relativistic corrections, which is power-ultravioletdivergent and needs subtraction [19]. This was an obstacle to evaluating relativistic corrections to the process. As the next step, QCD NLO corrections to the $e^{+} e^{-} \rightarrow J / \psi+\eta_{c}$ process were calculated. The $K$ factor for the QCD NLO prediction to that of LO in both $\alpha_{s}$ and $v$ was about a factor of $2[32,33]$. In spite of a large enhancement from the QCD NLO corrections, large discrepancy between theory and experiments still remained.
The only sector that had not been explored thoroughly was relativistic corrections to the process. The first prediction of the $K$ factor for the relativistic corrections was $2.0_{-1.1}^{+10.9}$ [19]. The main source for the huge uncertainty was from the relative-order- $v^{2}$ NRQCD matrix element. In principle, once the quarkonium color-singlet wave function is known by using a non-perturbative method such as lattice NRQCD $[48,49]$, one can compute the order- $v^{2}$ NRQCD matrix element, where power-ultraviolet-divergent contributions to the matrix element must be subtracted with dimensional regularization to make it consistent with the standard NRQCD factorization formalism. Because of this large subtraction, even the sign of the matrix element was uncertain by the time the prediction was made [19].

As a breakthrough, a new method to determine the color-singlet $S$-wave NRQCD matrix elements was introduced [30]. Employing the Cornell potential [50], which is a linear combination of the Coulomb potential and the linear potential, the authors computed the quarkonium color-singlet wave function [30,51]. Relative strength of the Coulomb potential compared to the linear potential was determined by making use of the lattice string tension [30]. Furthermore, they derived the relation, $\left\langle\boldsymbol{q}^{2 n}\right\rangle_{H}=\left\langle\boldsymbol{q}^{2}\right\rangle_{H}^{n}$ [30], which is the generalization of the Gremm-Kapustin relation [52], where $\left\langle\boldsymbol{q}^{2 n}\right\rangle$ is the ratio of the relative-order$v^{2 n}$ color-singlet matrix element to $\left\langle\mathcal{O}_{1}\right\rangle_{H}[30]$. The generalized Gremm-Kapustin relation made it possible for one to resum a class of relativistic corrections of color-singlet contributions to all orders in $v$ [30]. The resummation method was applied to improve the NRQCD factorization formula for the electromagnetic decay of the $J / \psi$. They determined the parameters of the Cornell potential model and the normalization of the quarkonium wave function by making use of the radial mass splittings of the $S$-wave states and electromagnetic decay widths of the $S$-wave states. As a result, the LO and order- $v^{2}$ NRQCD matrix elements of the $S$-wave quarkonium $H$ were determined [30]. In an updated analysis, they reported $\left\langle\mathcal{O}_{1}\right\rangle_{J / \psi}=0.440_{-0.055}^{+0.067} \mathrm{GeV}^{3}$ and $\left\langle\boldsymbol{q}^{2}\right\rangle_{J / \psi}=0.441_{-0.140}^{+0.140} \mathrm{GeV}^{2}$ [53]. This result cor- 
responds to $\left\langle v^{2}\right\rangle_{J / \psi}=0.225_{-0.088}^{+0.106}$, which is consistent with the naive estimate $v^{2} \approx 0.3$ within errors. This is the most reliable determination of the color-singlet NRQCD matrix elements for $J / \psi$ available so far [53].

The method of resummation [30] has been applied to resum a class of the relativistic corrections to the $e^{+} e^{-} \rightarrow J / \psi+\eta_{c}[31]$ in combination with the QCD NLO corrections in Ref. [32]. In the following updated study in Ref. [34], the authors reported the prediction $\sigma\left[e^{+} e^{-} \rightarrow J / \psi+\right.$ $\left.\eta_{c}\right]=17.6_{-6.3}^{+7.8} \mathrm{fb}$, which agrees with the experiments within errors. For more detailed descriptions of estimating theoretical uncertainties, we refer the readers to Ref. [34]. Therefore, in spite of large error bars, it seems fair to conclude that the long-standing puzzle between theory and experiments for the $e^{+} e^{-} \rightarrow J / \psi+\eta_{c}$ has been resolved [34].

Independently of the theoretical analysis in Ref. [34], a fixed-order relativistic corrections of relative-order $v^{2}$ to the cross section for $e^{+} e^{-} \rightarrow$ $J / \psi+\eta_{c}$ is given in Ref. [55], where the nonperturbative factors $\left\langle\mathcal{O}_{1}\right\rangle_{J / \psi}$ and $\left\langle\boldsymbol{q}^{2}\right\rangle_{J / \psi}$ are determined by making use of the measured values for $\Gamma\left[J / \psi \rightarrow e^{+} e^{-}\right], \Gamma\left[\eta_{c} \rightarrow \gamma \gamma\right]$, and $\Gamma[J / \psi \rightarrow$ $3 g]$ with the assumption $\left\langle\mathcal{O}_{1}\right\rangle_{J / \psi}\left\langle\boldsymbol{q}^{2}\right\rangle_{J / \psi}=$ $\left\langle\mathcal{O}_{1}\right\rangle_{\eta_{c}}\left\langle\boldsymbol{q}^{2}\right\rangle_{\eta_{c}}$. They obtained the cross section $20.04 \mathrm{fb}$ with $\left\langle\mathcal{O}_{1}\right\rangle_{J / \psi}=0.573 \mathrm{GeV}^{3}$ and $\left\langle\boldsymbol{q}^{2}\right\rangle_{J / \psi}=0.202 \mathrm{GeV}^{2}[55]$. The values for the nonperturbative factors yields $\left\langle v^{2}\right\rangle_{J / \psi} \approx 0.09$. It is smaller by about a factor of 2 than that obtained in Ref. [53], which is consistent with the naive estimate $v^{2} \approx 0.3$. We notice that the shortdistance coefficient of $\Gamma[J / \psi \rightarrow 3 g]$ at relativeorder $v^{2}$ is very large. This restricts the fit in Ref. [55] to have a small value for $\left\langle v^{2}\right\rangle_{J / \psi}$ [53]. Further applications to other exclusive doublecharmonium production processes are also available in Refs. [56,57].

\section{Inclusive charm production in bottomo- nia decays}

As described in Sec. 1, the earliest calculations of the widths of $P$-wave quarkonium states using perturbative QCD were plagued with infrared divergences [3,4]. In 1992, Bodwin, Braaten, and Lepage showed that the infrared divergences could be absorbed into the probability for the $Q \bar{Q}$ pair to be at the same point in a coloroctet state [2]. Based on the NRQCD factorization formalism, one can now carry out rigorous calculations of inclusive charm production from $\chi_{b J}$ decays. On the experimental side, $\chi_{b J}(1 P)$ and $\chi_{b J}(2 P)$ have been discovered. The only properties of these states that have been measured thus far are their masses and their radiative branching fractions into $\Upsilon(n S)$. The total widths of the $\chi_{b J}(n P)$ states have not been measured. Recent runs of the CLEO experiment at the $\Upsilon(2 S)$ and $\Upsilon(3 S)$ resonances have provided new data on the $\chi_{b J}(1 P)$ and $\chi_{b J}(2 P)$ states. The $B$-factory experiments BABAR and Belle can study the $\chi_{b J}(n P)$ states by using data samples of $\Upsilon(2 S)$ and $\Upsilon(3 S)$ provided by initial-state radiation. The Belle experiment has also accumulated data by running directly on the $\Upsilon(3 S)$ state. Therefore, it is necessary for theorists to present quantitative predictions for the $P$-wave bottomonium states that can be compared with forthcoming data.

The widths of all four states in a $P$-wave multiplet can be calculated by using the NRQCD factorization formula, once the two nonperturbative factors $\left\langle\mathcal{O}_{1}\right\rangle_{\chi_{b}}$ and $\left\langle\mathcal{O}_{8}\right\rangle_{\chi_{b}}$ have been determined. In Ref. [58], the authors computed the inclusive charm production rate in the $\chi_{b J}$ decay $\chi_{b J} \rightarrow c+X$. They also provided the momentum distributions of charmed hadrons produced in the process by making use of a parametrization for the charm fragmentation function fit to the data from $e^{+} e^{-}$collisions [59]. The predictions are dependent on the ratio $\rho_{8} \equiv m_{b}^{2}\left\langle\mathcal{O}_{8}\right\rangle_{\chi_{b}} /\left\langle\mathcal{O}_{1}\right\rangle_{\chi_{b}}$ that can be determined phenomenologically. Recently, the CLEO Collaboration measured the total production rates for the charm in $\chi_{b J}$ decays and the branching fractions for $\chi_{b J}(1 P, 2 P) \rightarrow D^{0} X$ with $p_{D^{0}}>2.5 \mathrm{GeV}$ [60]. The CLEO measurement indeed determined $\rho_{8}(1 P)=0.160_{-0.047}^{+0.071}$ and $\rho_{8}(2 P)=0.074_{-0.008}^{+0.010}[60]$. The joint fit for $1 P$ and $2 P$ gives $\rho_{8}=0.086_{-0.013}^{+0.009}[60]$.

As shown in Ref. [58], the branching fraction $\operatorname{Br}\left[\chi_{b J} \rightarrow c+X\right]$ must be particularly large for the $J=1$ state. The reason is that the $\chi_{b 1} \rightarrow c \bar{c} g$ 
is one of the LO contributions $\chi_{b 1} \rightarrow q \bar{q} g$ to hadronic decay of $\chi_{b 1}$, while the mode $\chi_{b J} \rightarrow c \bar{c} g$ is suppressed by order $\alpha_{s}$ compared to the leading contribution $\chi_{b J} \rightarrow g g$ for hadronic decays of $J=0$ and 2 states. Note that $\chi_{b 1} \rightarrow g g$ is forbidden by Yang's theorem. The measurement of the CLEO Collaboration also confirmed the large branching fractions of about $25 \%$ for both $\chi_{b 1}(1 P)$ and $\chi_{b 1}(2 P)$ [60], which is consistent with the prediction in Ref. [58] within errors.

Theoretical studies on the inclusive charm production has been extended to the $S$-wave bottomonium states in Refs. [61-64].

\section{Conclusions}

The discrepancy between the theoretical prediction and the CDF measurements at Run I and Run II of the Fermilab Tevatron for the prompt $S$-wave spin-triplet charmonium produced at large $p_{T}$ is embarrassing. To make the problem more complicated, there is a big discrepancy between the CDF data of Run I and those of Run II that has not been clearly understood, yet. In order to find any other contributions that may modify the theoretical prediction toward experimental results, efforts have been made in various manners. A few sources which contribute to the longitudinal polarization for the prompt $J / \psi$ and $\psi(2 S)$ are found. However, the complete analysis which includes all of these contributions and includes all the feeddowns that are contained in the data is not available, yet. In order to make a reliable prediction for the polarization with these new contributions, more accurate determination of various NRQCD matrix elements for the quarkonium production must be completed, which may not be an easy task.

The puzzle of the cross section for $e^{+} e^{-} \rightarrow$ $J / \psi+\eta_{c}$ at the $B$ factories has spurred rapid developments in heavy-quarkonium phenomenology. It led the first reliable estimate of the relative-order- $v^{2}$ color-singlet NRQCD matrix element for the $J / \psi$, that was uncertain even in the sign. A new method to resum relativistic corrections to a class of color-singlet contributions has been developed to determine the color-singlet NRQCD matrix elements for the $S$-wave heavy quarkonia more accurately. A recent computation of order- $\alpha_{s}$ corrections to the quarkonium electromagnetic current to all orders in $v$ revealed that the velocity expansion for approximate $J / \psi$ operator matrix elements converges very rapidly [54]. This supports the reliability of the resummation method. Being applied to the $e^{+} e^{-} \rightarrow J / \psi+\eta_{c}$ process, the resummation method for the relativistic corrections has resolved the puzzle in combination with large QCD corrections. The puzzle also motivated the proofs of the factorization theorems for exclusive two-body charmonium production in $B$-meson decay and $e^{+} e^{-}$annihilation to all orders in perturbation theory in quantum chromodynamics [65].

Resolution of the infrared-singularity problem in $P$-wave heavy-quarkonium decay into hadrons was the first successful theoretical application of the NRQCD factorization. However, the process has never been thoroughly investigated experimentally. A new measurement by the CLEO Collaboration of the inclusive charm production rate in $P$-wave bottomonium decay has provided useful information. The measurement helped us determine the ratio for the color-octet NRQCD matrix element to the color-singlet matrix element for the $P$-wave states accurately. On-going analysis by the CLEO Collaboration on the charmhadron momentum distribution may reveal the color-octet mechanism in $P$-wave bottomonium decay more clearly.

\section{REFERENCES}

1. G. T. Bodwin, E. Braaten and G. P. Lepage, Phys. Rev. D 51 (1995) 1125 [Erratum-ibid. D 55 (1997) 5853] [arXiv:hep-ph/9407339].

2. G. T. Bodwin, E. Braaten and G. P. Lepage, Phys. Rev. D 46 (1992) 1914 [arXiv:heplat/9205006].

3. R. Barbieri, R. Gatto and E. Remiddi, Phys. Lett. B 61 (1976) 465.

4. R. Barbieri, M. Caffo, R. Gatto and E. Remiddi, Phys. Lett. B 95 (1980) 93.

5. F. Abe et al. [CDF Collaboration], Phys. Rev. Lett. 79 (1997) 572.

6. F. Abe et al. [CDF Collaboration], Phys. Rev. Lett. 79 (1997) 578. 
7. E. Braaten and T. C. Yuan, Phys. Rev. Lett. 71 (1993) 1673 [arXiv:hep-ph/9303205].

8. E. Braaten and S. Fleming, Phys. Rev. Lett. 74 (1995) 3327 [arXiv:hep-ph/9411365].

9. P. L. Cho, M. B. Wise and S. P. Trivedi, Phys. Rev. D 51 (1995) 2039 [arXiv:hep$\mathrm{ph} / 9408352]$.

10. P. L. Cho and M. B. Wise, Phys. Lett. B 346 (1995) 129 [arXiv:hep-ph/9411303].

11. M. Beneke and I. Z. Rothstein, Phys. Lett. B 372 (1996) 157 [Erratum-ibid. B 389 (1996) 769] [arXiv:hep-ph/9509375].

12. A. K. Leibovich, Phys. Rev. D 56 (1997) 4412 [arXiv:hep-ph/9610381].

13. E. Braaten, B. A. Kniehl and J. Lee, Phys. Rev. D 62 (2000) 094005 [arXiv:hep$\mathrm{ph} / 9911436]$.

14. B. A. Kniehl and J. Lee, Phys. Rev. D 62 (2000) 114027 [arXiv:hep-ph/0007292].

15. A. A. Affolder et al. [CDF Collaboration], Phys. Rev. Lett. 85 (2000) 2886 [arXiv:hepex/0004027].

16. N. Brambilla et al. [Quarkonium Working Group], arXiv:hep-ph/0412158.

17. M. Bedjidian et al., arXiv:hep-ph/0311048.

18. K. Abe et al. [Belle Collaboration], Phys. Rev. Lett. 89 (2002) 142001 [arXiv:hepex/0205104].

19. E. Braaten and J. Lee, Phys. Rev. D 67 (2003) 054007 [Erratum-ibid. D 72 (2005) 099901] [arXiv:hep-ph/0211085].

20. K. Y. Liu, Z. G. He and K. T. Chao, Phys. Lett. B 557 (2003) 45 [arXiv:hep$\mathrm{ph} / 0211181]$.

21. B. Aubert et al. [BABAR Collaboration], Phys. Rev. D 72 (2005) 031101 [arXiv:hepex/0506062].

22. K. Abe et al. [Belle Collaboration], Phys. Rev. D 70 (2004) 071102 [arXiv:hep-ex/0407009].

23. See, for example, B. D. Yabsley, In the Proceedings of International Workshop on Charm Physics (Charm 2007), Ithaca, New York, 5-8 Aug 2007, pp 03 [arXiv:0712.3183 [hep-ex]].

24. G. T. Bodwin, J. Lee and E. Braaten, Phys. Rev. Lett. 90 (2003) 162001 [arXiv:hep$\mathrm{ph} / 0212181]$.

25. G. T. Bodwin, J. Lee and E. Braaten, Phys. Rev. D 67 (2003) 054023 [Erratum-ibid. D 72
(2005) 099904] [arXiv:hep-ph/0212352].

26. S. J. Brodsky, A. S. Goldhaber and J. Lee, Phys. Rev. Lett. 91 (2003) 112001 [arXiv:hep-ph/0305269].

27. A. E. Bondar and V. L. Chernyak, Phys. Lett. B 612 (2005) 215 [arXiv:hep-ph/0412335].

28. J. P. Ma and Z. G. Si, Phys. Rev. D 70 (2004) 074007 [arXiv:hep-ph/0405111].

29. G. T. Bodwin, D. Kang and J. Lee, Phys. Rev. D 74 (2006) 114028 [arXiv:hep$\mathrm{ph} / 0603185]$.

30. G. T. Bodwin, D. Kang and J. Lee, Phys. Rev. D 74 (2006) 014014 [arXiv:hep$\mathrm{ph} / 0603186]$.

31. G. T. Bodwin, D. Kang, T. Kim, J. Lee and C. Yu, AIP Conf. Proc. 892 (2007) 315 [arXiv:hep-ph/0611002].

32. Y. J. Zhang, Y. j. Gao and K. T. Chao, Phys. Rev. Lett. 96 (2006) 092001 [arXiv:hep$\mathrm{ph} / 0506076]$.

33. B. Gong and J. X. Wang, Phys. Rev. D 77 (2008) 054028 [arXiv:0712.4220 [hep-ph]].

34. G. T. Bodwin, J. Lee and C. Yu, Phys. Rev. D 77 (2008) 094018 [arXiv:0710.0995 [hep-ph]].

35. G. T. Bodwin, J. Lee and D. K. Sinclair, Phys. Rev. D 72 (2005) 014009 [arXiv:heplat/0503032].

36. A. Abulencia et al. [CDF Collaboration], Phys. Rev. Lett. 99 (2007) 132001 [arXiv:0704.0638 [hep-ex]].

37. E. Braaten and J. Lee, Phys. Rev. D 63 (2001) 071501 [arXiv:hep-ph/0012244].

38. E. Braaten, S. Fleming and A. K. Leibovich, Phys. Rev. D 63 (2001) 094006 [arXiv:hepph/0008091].

39. D. E. Acosta et al. [CDF Collaboration], Phys. Rev. Lett. 88 (2002) 161802.

40. C. N. Brown et al. [FNAL E866 Collaboration and NuSea Collaboration], Phys. Rev. Lett. 86 (2001) 2529 [arXiv:hep-ex/0011030].

41. [D0 Collaboration], arXiv:0804.2799 [hep-ex].

42. J. Campbell, F. Maltoni and F. Tramontano, Phys. Rev. Lett. 98 (2007) 252002 [arXiv:hep-ph/0703113].

43. P. Artoisenet, J. P. Lansberg and F. Maltoni, Phys. Lett. B 653 (2007) 60 [arXiv:hep$\mathrm{ph} / 0703129]$.

44. P. Artoisenet, J. Campbell, J. P. Lans- 
berg, F. Maltoni and F. Tramontano, arXiv:0806.3282 [hep-ph].

45. B. Gong and J. X. Wang, arXiv:0802.3727 [hep-ph].

46. B. Gong and J. X. Wang, arXiv:0805.2469 [hep-ph].

47. B. Gong, X. Q. Li and J. X. Wang, arXiv:0805.4751 [hep-ph].

48. G. T. Bodwin, D. K. Sinclair and S. Kim, Phys. Rev. Lett. 77 (1996) 2376 [arXiv:heplat/9605023].

49. G. T. Bodwin, D. K. Sinclair and S. Kim, Phys. Rev. D 65 (2002) 054504 [arXiv:heplat/0107011].

50. E. Eichten, K. Gottfried, T. Kinoshita, K. D. Lane and T. M. Yan, Phys. Rev. D 17 (1978) 3090 [Erratum-ibid. D 21 (1980) 313].

51. H. S. Chung, J. Lee and D. Kang, J. Korean Phys. Soc. 52 (2008) 1151 [arXiv:0803.3116 [hep-ph]].

52. M. Gremm and A. Kapustin, Phys. Lett. B 407 (1997) 323 [arXiv:hep-ph/9701353].

53. G. T. Bodwin, H. S. Chung, D. Kang, J. Lee and C. Yu, Phys. Rev. D 77 (2008) 094017 [arXiv:0710.0994 [hep-ph]].

54. G. T. Bodwin, H. S. Chung, J. Lee and C. Yu, arXiv:0807.2634 [hep-ph].

55. Z. G. He, Y. Fan and K. T. Chao, Phys. Rev. D 75 (2007) 074011 [arXiv:hep-ph/0702239].

56. Y. J. Zhang, Talk given at the 5th International workshop on heavy quarkonium 2007 (QWG 2007), Hamburg, Germany, 17-20 Oct 2007.

57. Y. J. Zhang, Y. Q. Ma and K. T. Chao, arXiv:0802.3655 [hep-ph].

58. G. T. Bodwin, E. Braaten, D. Kang and J. Lee, Phys. Rev. D 76 (2007) 054001 [arXiv:0704.2599 [hep-ph]].

59. R. Seuster et al. [Belle Collaboration], Phys. Rev. D 73 (2006) 032002 [arXiv:hepex/0506068].

60. R. A. Briere et al. [CLEO Collaboration], arXiv:0807.3757 [hep-ex].

61. D. Kang, T. Kim, J. Lee and C. Yu, Phys. Rev. D 76 (2007) 114018 [arXiv:0707.4056 [hep-ph]].

62. H. S. Chung, T. Kim and J. Lee, arXiv:0805.1989 [hep-ph].
63. Y. J. Zhang and K. T. Chao, arXiv:0808.2985 [hep-ph].

64. G. Hao, C. F. Qiao and P. Sun, Phys. Rev. D 76 (2007) 125013 [arXiv:0710.3339 [hep-ph]].

65. G. T. Bodwin, X. Garcia i Tormo and J. Lee, arXiv:0805.3876 [hep-ph], to appear in Phys. Rev. Lett. 